\title{
Nanoprobe-Based Affinity Mass Spectrometry for Selected Protein Profiling in Human Plasma
}

\author{
Po-Hung Chou,,t, Shu-Hua Chen,t,‡ Hsin-Kai Liao, † Po-Chiao Lin,t Gour-Rong Her,‡

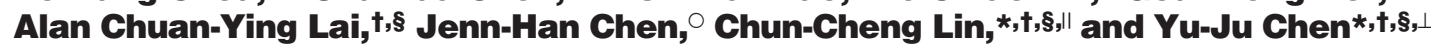

\begin{abstract}
Institute of Chemistry and Genomic Research Center, Chemical Biology and Molecular Biophysics, Taiwan International Graduate Program, Academia Sinica, Taipei 115, Taiwan, Department of Chemistry and Institute of Biochemical Science, College of Life Science, National Taiwan University, Taipei 106, Taiwan, Department of Applied Chemistry, National Chiao Tung University, Hsinchu 300, Taiwan, Department of Molecular Biology, National Chung-Hsing University, Taichun 402, Taiwan, and School of Dentistry, National Defense University, Taipei 114, Taiwan
\end{abstract}

In recent decades, magnetic nanoparticles have emerged as a promising new platform in biomedical applications, particularly bioseparations. We have developed an immunoassay using antibody-conjugated magnetic nanoparticles as an efficient affinity probe to simultaneously preconcentrate and isolate targeted antigens from biological media. We combined this probe with matrix-assisted laser desorption/ionization time-of-flight mass spectrometry (MALDI MS) to profile proteins in diluted human plasma. The nanoparticles were designed to detect several disease-associated proteins and could be used directly in MALDI MS without an elution step, thereby facilitating multiple antigen screening and the characterization of antigen variants. Plasma antigens bound rapidly $(\sim 10$ $\mathrm{min})$ to the antibody-conjugated nanoparticles, allowing the assay to be performed within $20 \mathrm{~min}$. With sensitivity of detection in the femtomole range, the nanoscale immunoassay is superior to assays using microscale particles. We applied our method to comparative protein profiling of patients with gastric cancer and healthy individuals and found differential protein expression levels associated with the disease as well as individuals. Given the flexibility of manipulating functional groups on the nanoprobes, their low cost, robustness, and simplicity of the assay, our approach shows promise for targeted proteome profiling in clinical settings.

The completion of the human genome project has catalyzed advances in proteomics to investigate cellular function at the protein level. Particularly, increasingly sophisticated techniques have been rapidly developed for discovering disease biomarkers via large-scale differential profiling. ${ }^{1,2}$ The recognition that most

\footnotetext{
* To whom the correspondence should be addressed. E-mail: yjchen@ chem.sinica.edu.tw; cclin@chem.sinica.edu.tw. Fax: +886-2-2783-1237.

$\dagger$ Institute of Chemistry and Genomic Research Center, Academia Sinica.

₹ Department of Chemistry, National Taiwan University.

$\S$ Chemical Biology and Molecular Biophysics, Taiwan International Graduate Program, Academia Sinica.

" Department of Applied Chemistry, National Chiao Tung University.

${ }^{\perp}$ Department of Molecular Biology, National Chung-Hsing University.

- School of Dentistry, National Defense University.

(1) Kantor, A. B.; Wang, W.; Lin, H.; Govindarajan, H.; Anderle, M.; Perrone, A.; Becker, C. Clin. Immunol. 2004, 111, 186-195.
}

diseases induce a specific pattern of change in proteomic microenvironments indicates important clinical implications on the early detection and progression of disease. ${ }^{3,4}$ Although plasma, urine, and saliva are readily available samples whose protein content reflects the environment encountered by the blood during its journey through tissues and the circulatory system, ${ }^{5}$ yet body fluid-derived proteomes are complex, with a wide and dynamic range in protein abundance that imposes extreme analytical difficulties for medical studies or clinical diagnoses. With the advent of a growing number of candidate protein biomarkers for disease diagnosis, the development of sensitive techniques with great potential to monitor disease onset is urgently needed for the next phase of targeted proteomics.

The detection and diagnosis of disease in the clinical setting primarily depends on immunoassays based on antibody-antigen interactions. The most widely used of all the methods, enzymelinked immunosorbent assay (ELISA), offers both specificity and sensitivity. Alternatively, protein chip-based approaches are increasingly used in clinical diagnosis, because the array format can be easily adapted to miniaturization, multiplexing, and high throughput. ${ }^{6-12}$ However, these traditional immunological methods are inconvenient and time-consuming because enzymes or fluorescent reagents have to be labeled. Fluorescence measurements also may have high background, leading to false positives, and produce photobleaching, leading to false negatives. ${ }^{13}$

(2) Petricoin, E.; Wulfkuhle, J.; Espina, V.; Liotta, L. A. J. Proteome Res. 2004, 3, 209-217.

(3) Liotta, L. A.; Kohn, E. C. Nature 2001, 411, 375-379.

(4) Hanash, S. Nature 2003, 422, 226-232.

(5) Anderson, N. L.; Anderson, N. G. Mol. Cell. Proteomics 2002, 1, 845-867.

(6) Templin, M. F.; Stoll, D.; Schrenk, M.; Traub, P. C.; Vohringer, C. F.; Joos, T. O. Trends Biotechnol. 2002, 20, 160-166.

(7) Wilson, D. S.; Nock, S. Curr. Opin. Chem. Biol. 2002, 6, 81-85.

(8) Nedelkov, D.; Nelson, R. W. J. Mol. Recognit. 2003, 16, 15-19.

(9) MacBeath, G.; Schreiber, S. L. Science 2000, 289, 1760-1763.

(10) Haab, B. B.; Dunham, M. J.; Brown, P. O. Genome Biol. 2001, 2, RESEARCH0004.

(11) Robinson, W. H.; DiGennaro, C.; Hueber, W.; Haab, B. B.; Kamachi, M.; Dean, E. J.; Fournel, S.; Fong, D.; Genovese, M. C.; de Vegvar, H. E.; Skriner K.; Hirschberg, D. L.; Morris, R. I.; Muller, S.; Pruijn, G. J.; van Venrooij, W. J.; Smolen, J. S.; Brown, P. O.; Steinman, L.; Utz, P. J. Nat. Med. 2002, $8,295-301$.

(12) Zhou, H.; Bouwman, K.; Schotanus, M.; Verweij, C.; Marrero, J. A.; Dillon, D.; Costa, J.; Lizardi, P.; Haab, B. B. Genome Biol. 2004, 5, R28.

(13) Graham, D. L.; Ferreira, H. A.; Freitas, P. P. Trends Biotechnol. 2004, 22, 455-462.

10.1021/ac050655o CCC: $\$ 30.25$ @ 2005 American Chemical Society Published on Web 08/16/2005 
Recent developments in mass spectrometry have greatly expanded the possibility of characterizing unknown proteins in proteomic research. Mass spectrometry is especially suitable for the direct detection of proteins (i.e., on the probe), which enhances specificity without the use of fluorescent or radioactive labels; this approach offers greater flexibility in the selection of bioactive probes. Among these developments, matrix-assisted laser desorption/ionization time-of-flight mass spectrometry (MALDITOF MS) has become one of the primary techniques for protein identification due to its high sensitivity, tolerance to impurities, and high speed. ${ }^{14}$ Despite these advantages, the simultaneous characterization of hundreds to thousands of proteins in complex media still remains a challenge due to the suppression effect. ${ }^{15}$ Recently, surface-enhanced laser desorption/ionization, has evolved rapidly as a new frontier for biomarker discovery and clinical diagnoses based on proteomic pattern analysis. ${ }^{2}$ Despite its advantages of high sensitivity and high throughput, the pattern recognition platform unfortunately suffers from laboratory-tolaboratory variance due to differences in sample handling and analysis software. ${ }^{16}$

As an alternative to the above approaches, MALDI MS can be combined with a biologically active probe to rapidly and specifically target proteins of interest. This targeted approach can accelerate research for class-specific proteins or biomarkers. ${ }^{17-21}$ Several analytical affinity capture techniques have been developed in the field of biological mass spectrometry. The research group of Hutchens and Yip was one of first to demonstrate MS-based affinity capture by immobilization of "bait" DNA on agarose beads for direct MALDI MS analysis of targeted proteins from complex biofluids. ${ }^{22}$ The concept was further tailored by Nelson and coworkers to develop a mass spectrometric immunoassay. ${ }^{23}$ They used affinity pipet tips to selectively retrieve proteins from biological solutions, demonstrating high-throughput quantitative protein analysis as well as screening of heterogeneous glycan structures in plasma proteins. ${ }^{24,25}$ Variations of the biologically active probe for affinity mass spectrometry include the assay of direct desorption/ionization on silicon ${ }^{26,27}$ and self-assembled monolayers..$^{28,29}$ Despite the rapid evolution of efficient chip-based or microbead-based assays for biomedical research, protein chip

(14) Karas, M.; Hillenkamp, F. Anal. Chem. 1988, 60, 2299-2301.

(15) Wulfkuhle, J. D.; Liotta, L. A.; Petricoin, E. F. Nat. Rev. Cancer 2003, 3, 267-275.

(16) Diamandis, E. P. Mol. Cell. Proteomics 2004, 3, 367-378.

(17) Bundy, J. L.; Fenselau, C. Anal. Chem. 2001, 73, 751-757.

(18) Min, D. H.; Tang, W. J.; Mrksich, M. Nat. Biotechnol. 2004, 22, 717-723.

(19) Warren, E. N.; Elms, P. J.; Parker, C. E.; Borchers, C. H. Anal. Chem. 2004, 76, 4082-4092.

(20) Zhang, Y.; Wang, X.; Shan, W.; Wu, B.; Fan, H.; Yu, X.; Tang, Y.; Yang, P. Angew. Chem., Int. Ed. 2005, 44, 615-617.

(21) Chen, Y. J.; Chen, S. H.; Chien, Y. Y.; Chang, Y. W.; Liao, H. K.; Chang, C. Y.; Jan, M. D.; Wang, K. T.; Lin, C. C. ChemBioChem 2005, 6, 1169-1173.

(22) Hutchens, T. W.; Yip, T. T. Rapid Commun. Mass Spectrom. 1993, 7, 576580.

(23) Nelson, R. W.; Krone, J. R.; Bieber, A. L.; Williams, P. Anal. Chem. 1995, 67, 1153-1158.

(24) Nedelkov, D.; Tubbs, K. A.; Niederkofler, E. E.; Kiernan, U. A.; Nelson, R. W. Anal. Chem. 2004, 76, 1733-1737.

(25) Kiernan, U. A.; Nedelkov, D.; Tubbs, K. A.; Niederkofler, E. E.; Nelson, R. W. Proteomics 2004, 4, 1825-1829.

(26) Wei, J.; Buriak, J. M.; Siuzdak, G. Nature 1999, 399, 243-246.

(27) Zou, H.; Zhang, Q.; Guo, Z.; Guo, B.; Zhang, Q.; Chen, X. Angew. Chem., Int. Ed. 2002, 41, 646-648.

(28) Brockman, A. H.; Orlando, R. Anal. Chem. 1995, 67, 4581-4585.

(29) Su, J.; Mrksich, M. Angew. Chem., Int. Ed. 2002, 41, 4715-4718. technologies face two main technical challenges. First, the physical and chemical properties of the chip surface may denature/alter the native three-dimensional structure of proteins, raising the possibility of disrupted bait-target protein interactions. ${ }^{19}$ Second, the requirement of specialized immobilization chemistry for surface engineering or specialized instruments limit the general application of these protein assay technologies in the general scientific community.

Recently, the development of biomolecule conjugated nanomaterials has shown promising applications in various biology systems. ${ }^{30-33}$ When affinity molecules are coupled to such nanoparticles (NPs), they can function as sensitive biosensors ${ }^{34}$ that show superior separation capabilities compared with microbeads. ${ }^{35,36}$ Moreover, there are several advantages of using NPs as ligand carriers. ${ }^{37,38}$ First, there are simple and facile means of anchoring molecules to the nanoscale surface through chemical functionalization to form versatile and covalently stable conjugates with the capabilities to specifically interact with targeted biomolecules. ${ }^{31}$ Second, the large surface area-to-volume ratio and the globular shape of an NP allow multivalent and three-dimensional interactions between ligands and receptors. ${ }^{39}$ Finally, compared with conventional microarray methods using planar solid supports, functionalized NPs can be used as "water-soluble" probes in biological assays in solution. In principle, these "suspension arrays" should improve assay homogeneity and decrease assay time due to radial diffusion without planar hindrance.

Advances in nanotechnology have extended molecular diagnostics to the nanoscale. New detection methods have been developed based on nanosized labels, such as quantum dots and fluorescent NPs, which are coupled to specific binding molecules such as antibodies and nucleic acids to detect specific biomarkers. A method combining a time-resolved immunofluorometric assay with NP was developed and used to detect free prostate-specific antigen (PSA-F) at high sensitivity. ${ }^{40} \mathrm{As}$ a consequence of the high density of antibodies covalently linked to the surface of NPs, they demonstrated improved rate constants for the association of analytes with multivalent antibody-conjugated NPs, thereby contributing to an extremely sensitive immunoassay. ${ }^{40-42}$ In another example, Mirkin et al. used oligonucleotide-modified gold NPs

(30) Michalet, X.; Pinaud, F. F.; Bentolila, L. A.; Tsay, J. M.; Doose, S.; Li, J. J.; Sundaresan, G.; Wu, A. M.; Gambhir, S. S.; Weiss, S. Science 2005, 307, $538-544$.

(31) Katz, E.; Willner, I. Angew. Chem., Int. Ed. Engl. 2004, 43, 6042-6108.

(32) Park, S.; Lim, J. H.; Chung, S. W.; Mirkin, C. A. Science 2004, 303, 348351.

(33) Boal, A. K.; Ilhan, F.; DeRouchey, J. E.; Thurn-Albrecht, T.; Russell, T. P.; Rotello, V. M. Nature 2000, 404, 746-748.

(34) Perez, J. M.; Josephson, L.; Weissleder, R. ChemBioChem 2004, 5, 261264.

(35) Xu, C.; Xu, K.; Gu, H.; Zhong, X.; Guo, Z.; Zheng, R.; Zhang, X.; Xu, B. J. Am. Chem. Soc. 2004, 126, 3392-3393.

(36) Gu, H.; Ho, P. L.; Tsang, K. W.; Wang, L.; Xu, B. J. Am. Chem. Soc. 2003, 125, 15702-15703.

(37) Hayat, M. A. Colloidal Gold: Principles, Methods and Applications; Academic Press: New York, 1989.

(38) Hamley, I. W. Angew. Chem., Int. Ed. 2003, 42, 1692-1712.

(39) Lin, C. C.; Yeh, Y. C.; Yang, C. Y.; Chen, G. F.; Chen, Y. C.; Wu, Y. C.; Chen, C. C. Chem. Commun. 2003, 23, 2920-2921.

(40) Soukka, T.; Paukkunen, J.; Harma, H.; Lonnberg, S.; Lindroos, H.; Lovgren, T. Clin. Chem. 2001, 47, 1269-1278.

(41) Soukka, T.; Harma, H.; Paukkunen, J.; Lovgren, T. Anal. Chem. 2001, 73, 2254-2260.

(42) Soukka, T.; Antonen, K.; Harma, H.; Pelkkikangas, A. M.; Huhtinen, P.; Lovgren, T. Clin. Chim. Acta 2003, 328, 45-58. 
and antibody-modified magnetic microparticles to detect PSA at low-attomolar levels. ${ }^{43}$ Together, these studies demonstrate that the incorporation of nanotechnology into the design of biological assays can significantly improve sensitivity and reproducibility.

Since the mid-1970s, there has been increasing development of magnetic beads for applications in bioscience and medicine. ${ }^{44,45}$ The most documented and extensive application of magnetic nanoparticles (mNPs) is to achieve separation of analytes and thus shorten the total sample-processing time. In addition to the utility of mNPs for sample cleanup and preconcentration in MALDI MS, surface-functionalized mNPs permit selective extraction of pathogenic bacteria ${ }^{46,47}$ and aptamer-bound peptides. ${ }^{48}$

In this report, we present a nanoprobe-based immunoassay that combines antibody-conjugated mNPs with mass spectrometry detection. We have previously demonstrated that the nanoprobebased affinity mass spectrometry can be an effective method for simultaneous separation and enrichment of target proteins and then protein identification and epitope mapping by MALDI-TOF MS. ${ }^{21}$ The key component in the current assay is the use of mNPs, which surfaces are covalently encapsulated with specific antibodies that serve as "molecular probe" to isolate disease-related antigens from human plasma. After the immunoaffinity-capture process, the nanoprobe can be directly analyzed by MALDI MS. To illustrate the selectivity and sensitivity of the nanoprobe-based immunoassay, we selected two model proteins, C-reactive protein (CRP) and serum amyloid $\mathrm{P}$ component (SAP), with mean concentrations of $0.8^{49}$ and $30-45 \mathrm{mg} / \mathrm{L}$, respectively, in healthy subjects. ${ }^{50}$ These two acute-phase proteins serve as important biomarkers in many fatal diseases. ${ }^{49,51}$ The assay procedure is simple, requires minimal sample handling, and can be completed in $20 \mathrm{~min}$. With optimization, the assay can detect these diseaseassociated proteins even in highly diluted human blood. Finally, the assay was applied to comparative protein profiling of patients and healthy individuals, which also demonstrated the capability to simultaneously screen posttranslational modifications of targeted plasma proteins.

\section{EXPERIMENTAL SECTION}

Materials. Cytochrome $c$, myoglobin, enolase, and sinapinic acid (SA) were purchased from Sigma-Aldrich (Mississauga, ON, Canada). SAP and CRP were obtained from Calbiochem (San Diego, CA). Polyclonal anti-SAP was purchased from DakoCytomation (Carpinteria, CA). Monoclonal anti-CRP was purchased from Biodesign (Kennebunk, ME). The magnetic Separator was from Qiagen (Valencia, CA).

Synthesis of Antibody-Conjugated Magnetic Nanoparticles. Iron oxide nanoparticles $\left(\mathrm{Fe}_{3} \mathrm{O}_{4}\right)$ were synthesized by

(43) Nam, J. M.; Thaxton, C. S.; Mirkin, C. A. Science 2003, 301, 1884-1886.

(44) Mosbach, K.; Andersson, L. Nature 1977, 270, 259-261.

(45) Shinkai, M. J. Biosci. Bioeng. 2002, 94, 606-613.

(46) Lin, Y. S.; Tsai, P. J.; Weng, M. F.; Chen, Y. C. Anal. Chem. 2005, 77, $1753-1760$.

(47) Ho, K. C.; Tsai, P. J.; Lin, Y. S.; Chen, Y. C. Anal. Chem. 2004, 76, 71627168.

(48) Turney, K.; Drake, T. J.; Smith, J. E.; Tan, W.; Harrison, W. W. Rapid Commun. Mass Spectrom. 2004, 18, 2367-2374.

(49) Pepys, M. B.; Hirschfield, G. M. J. Clin. Invest. 2003, 111, 1805-1812.

(50) Pepys, M. B.; Dash, A. C.; Markham, R. E.; Thomas, H. C.; Williams, B. D.; Petrie, A. Clin. Exp. Immunol. 1978, 32, 119-124.

(51) Pepys, M. B.; Rademacher, T. W.; Amatayakul-Chantler, S.; Williams, P.; Noble, G. E.; Hutchinson, W. L.; Hawkins, P. N.; Nelson, S. R.; Gallimore, J. R.; Herbert, J.; et al. Proc. Natl. Acad. Sci. U.S.A. 1994, 91, 5602-5606. coprecipitation using $\mathrm{FeCl}_{2}$ and $\mathrm{FeCl}_{3}$ under basic conditions. ${ }^{52}$ During preparation, the desired "core" mNPs were treated with tetraethyl orthosilicate to create the silica-coated surface. Subsequently, (3-aminopropyl) trimethoxysilane was added for aminosilane modification to give mNPs.

The antibody of interest was covalently linked to the mNPs surface through the cross-linker bis ( $N$-hydroxysuccinimide ester) (NHS-ester; DSS). In brief, aminosilane-modified mNPs $(0.5 \mathrm{mg})$ were dissolved in DMSO $(0.5 \mathrm{~mL})$ and immediately incubated with DSS solution for $1 \mathrm{~h}$ at room temperature. This solution was then dried and resuspended with either anti-SAP solution $(8.1 \mathrm{mg} /$ $\mathrm{mL}, 25 \mu \mathrm{L})$ or anti-CRP solution $(15.4 \mathrm{mg} / \mathrm{mL}, 15 \mu \mathrm{L})$. The mixture was incubated at $4{ }^{\circ} \mathrm{C}$ for overnight. The cross-linker is active for primary amines and thus can bridge between the aminosilanemodified mNPs and antibodies. Finally, the antibody-conjugated mNPs products were magnetically separated and extensively washed with PBS (0.1 M, pH 7.4) to remove excess reactants. The final product was dried and stored at $4{ }^{\circ} \mathrm{C}$ for further use.

To compare with magnetic microbeads, commercially available magnetic microbeads (Dynal Biotech, $2.8 \mu \mathrm{m}$ ) were conjugated with antibody as described above.

Immunoaffinity Capture of Antigens. For the proof-ofprinciple experiment, $10 \mu \mathrm{g}$ of mNPs was immersed in $60 \mu \mathrm{L}$ of PBS (pH 7.4), containing $0.5 \mu \mathrm{M}$ myoglobin, $0.1 \mu \mathrm{M}$ CRP, 0.67 $\mu \mathrm{M}$ SAP, and $2.1 \mu \mathrm{M}$ enolase for $60 \mathrm{~min}$ at room temperature. Unbound proteins were removed by isolating the mNPs using a magnetic separator; the mNPs were then washed four times with $100 \mu \mathrm{L}$ of $25 \mathrm{mM}$ ammonium bicarbonate. For subsequent MALDI MS analysis, the mNPs were directly mixed with $1-2 \mu \mathrm{L}$ of MALDI matrix SA $(10 \mathrm{mg}$ of SA dissolved in $1 \mathrm{~mL}$ of solution containing $50 \%$ acetonitrile, $50 \%$ water, and $0.1 \%$ trifluoroacetic acid), spotted onto the sample plate, air-dried, and analyzed. For human plasma analyses, aliquots $(5-20 \mu \mathrm{L})$ of plasma, diluted in PBS ( $\mathrm{pH} 7.4$ ), were mixed with mNPs (conjugated with either anti-SAP or anti-CRP) and subjected to the same immunoaffinity reaction and MALDI MS analysis.

To evaluate the effect of incubation time, $1-\mu \mathrm{L}$ aliquots of 50 $\mu \mathrm{L}(40 \mathrm{ng} / \mu \mathrm{L} \mathrm{SAP})$ samples from supernatant were immediately transferred to the sample plate at different times: $0,3,10,30$, and $60 \mathrm{~min}$. Each sample was then applied with $0.5 \mu \mathrm{L}$ of MALDI matrix and subsequently analyzed by MALDI MS.

Mass Spectrometry. All mass spectra were acquired by using a MALDI-TOF mass spectrometer Voyager DE-STR (PerSeptive Biosystems) equipped with a 337-nm nitrogen laser. The spectra were recorded in the linear mode using an accelerating voltage of $25 \mathrm{kV}$, a $90 \%$ grid voltage, $0.3 \%$ guide wire voltage, 650 -ns delay time, and a low-mass gate of $5000 \mathrm{Da}$. External mass calibration was usually applied based on a mixture of two reference proteins [cytochrome $c(\mathrm{MW}=12361)$ and myoglobin $(\mathrm{MW}=16952)$ ] covering the $m / z$ range of $5-80 \mathrm{kDa}$. A typical mass spectrum was obtained by averaging 250 laser shots followed by noise reduction and Gaussian smoothing using Data-Explorer software (Applied Biosystems, Foster City, CA).

Plasma Samples. Plasma samples from 4 patients with gastric cancer and 20 healthy controls were obtained with informed consent from the Department of General Surgery, Tri-Services

(52) Kang, Y. S.; Risbud, S.; Rabolt, J. F.; Stroeve, P. Chem. Mater. 1996, 8 , 2209-2211. 


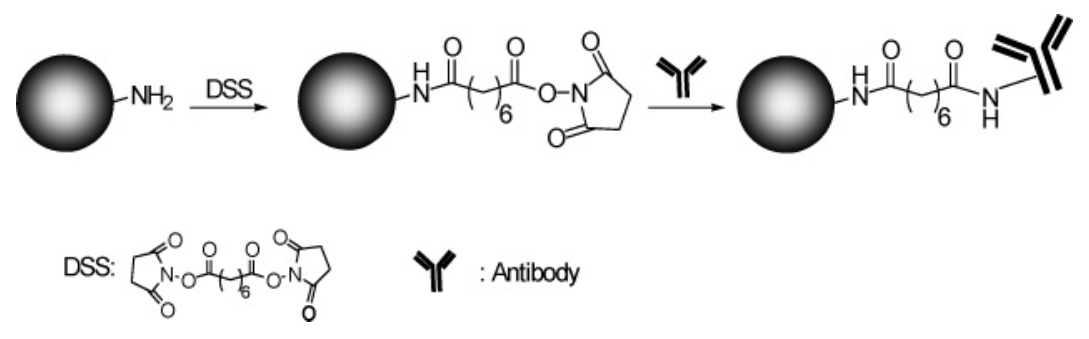

General Hospital, Taipei, Taiwan. The procedure was approved by the Review Boards of Tri-Service General Hospital, National Defense Medical Center. Plasma levels of CRP were measured by the latex particle-enhanced immunonephelometric assay using a nephelometer (Dade Behring, Marburg, Germany). ${ }^{53}$ SAS 8.0 statistical software (SAS Institute GmbH, Heidelberg, Germany) was used for statistical analysis. The Wilcoxon rank sum test was used to compare CRP levels between healthy donors and patients with gastric cancer.

\section{RESULTS AND DISCUSSION}

Preparation and Characterization of Nanoparticles. The synthesis of antibody-conjugated mNPs is illustrated in Scheme 1. The superparamagnetic nanoparticles $\left(\mathrm{Fe}_{3} \mathrm{O}_{4}\right)$ were synthesized as reported by Stroeve et al. ${ }^{52}$ Nanoprobe stability and specific activity are important parameters for the engineering of an optimal nanoparticle-antibody immunoassay. ${ }^{54}$ To develop more stable antibody-nanoparticle bioconjugates, the antibodies of interest (anti-SAP and anti-CRP) were covalently conjugated to the aminosilane-modified mNPs through the bifunctionally amineactive cross-linker (DSS) (Scheme 1) ${ }^{52}$ Figure 1A shows a
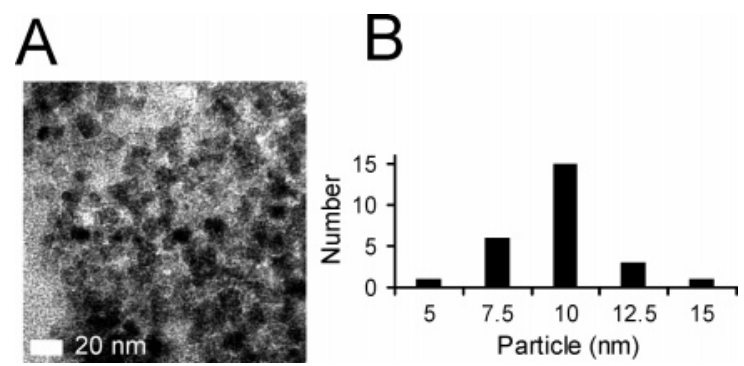

Figure 1. Particle size analysis by transmission electron microscopy (TEM). (A) A TEM image of the iron oxide core. (B) The size distribution histogram of the particles shows an average diameter of $9.7 \mathrm{~nm}$.

transmission electron micrograph of the antibody-conjugated mNPs. Small particle size can reduce steric hindrance and thus improve specific activity. ${ }^{2}$ The size distribution histogram of the mNPs (Figure 1B) indicates that the diameter of iron oxide "core" ranges from 5 to $15 \mathrm{~nm}$, with an average diameter of $\sim 9.7 \mathrm{~nm} .{ }^{9}$ Choosing particles below $15 \mathrm{~nm}$ in diameter ensures their superparamagnetism, stability, and dispersion upon removal of

(53) Juan, H. F.; Chen, J. H.; Hsu, W. T.; Huang, S. C.; Chen, S. T.; Yi-Chung Lin, J.; Chang, Y. W.; Chiang, C. Y.; Wen, L. L.; Chan, D. C.; Liu, Y. C.; Chen, Y. J. Proteomics 2004, 4, 2766-2775.

(54) Bilkova, Z.; Castagna, A.; Zanusso, G.; Farinazzo, A.; Monaco, S.; Damoc, E.; Przybylski, M.; Benes, M.; Lenfeld, J.; Viovy, J. L.; Righetti, P. G. Proteomics 2005, 5, 639-647. the magnetic field. ${ }^{55}$ Approximately $46 \mu \mathrm{g}$ (320 pmol) of anti-SAP was immobilized on the surface of $1.0 \mathrm{mg}$ of mNPs, as determined using the BCA protein assay. Similarly, $\sim 9.8 \mu \mathrm{g}$ ( $66 \mathrm{pmol}$ ) of monoclonal anti-CRP was immobilized. These antibody-nanoparticle bioconjugates could be stored in $\mathrm{PBS}, \mathrm{pH} 7.4$, at $4{ }^{\circ} \mathrm{C}$ for at least six months without decomposition.

Proof-of-Principle Experiment. To demonstrate our strategy, aliquots of functionalized mNPs were incubated with biological medium containing the targeted antigen. After the immunoaffinity interaction, the antigen-nanoparticle complexes were separated using a magnet, and nonantigenic proteins and interfering impurities were subsequently removed by a series of washes, abrogating the need for purification and desalting. Finally, the nanoparticles were directly mixed with matrix for MALDI MS analyses. To mimic a complex biological medium, we prepared a protein pool in $60 \mu \mathrm{L}$ of PBS (0.01 M, pH 7.4) composed of antigenic proteins (SAP, 20\% molar fraction and CRP, 3\% molar fraction) and two other "nonantigenic" proteins, myoglobin (Myo, 15\%) and enolase (Eno, 62\%). The abundance of the antigenic proteins was purposely reduced to test the extraction efficiency of the targeted protein.

Prior to affinity extraction, as shown in Figure 2A, the MALDI spectrum of the protein mixture shows the complexity of the mixture, in which the targeted antigen, CRP, was not observed due to its low abundance ( $3 \%$ molar fraction) and the ion suppression effect. ${ }^{56}$ Suppression effects are commonly observed in MALDI MS due to the presence of salts, buffer, or other more abundant species in complex biological media. ${ }^{57}$ The suppression effect can result in reduced signal intensity or even disappearance of the targeted analyte.

After affinity extraction, the MALDI spectrum in Figure 2B reveals the specificity of nanoscale immunoassay, where $\mathrm{CRP}$ was selectively concentrated and detected with an excellent signal-tonoise ratio of 822 . No background peak between $m / z 5000$ and 50000 was observed in control experiments before the addition of the protein mixture, showing no "chemical noise" arising from the antibody-conjugated mNPs. The absence of other abundant proteins in Figure 2B excluded nonspecific binding arising from electrostatic attraction or hydrogen bonding. The use of mNPs as a MALDI substrate overcomes the suppression effect because the particles are washed extensively to remove salt and abundant/ nonantigenic proteins from the biological sample. The clean mass spectrum demonstrates the advantages of nanoprobe-based affinity extraction in providing simultaneous protein isolation, enrichment,

(55) Tartaj, P.; Morales, M. P.; Veintemillas-Verdaguer, S.; González-Carreño, T.; Serna, C. J. J. Phys. D: Appl. Phys. 2003, 36, R182-197.

(56) Krause, E.; Wenschuh, H.; Jungblut, P. R. Anal. Chem. 1999, 71, 41604165.

(57) Knochenmuss, R.; Zenobi, R. Chem. Rev. 2003, 103, 441-452. 


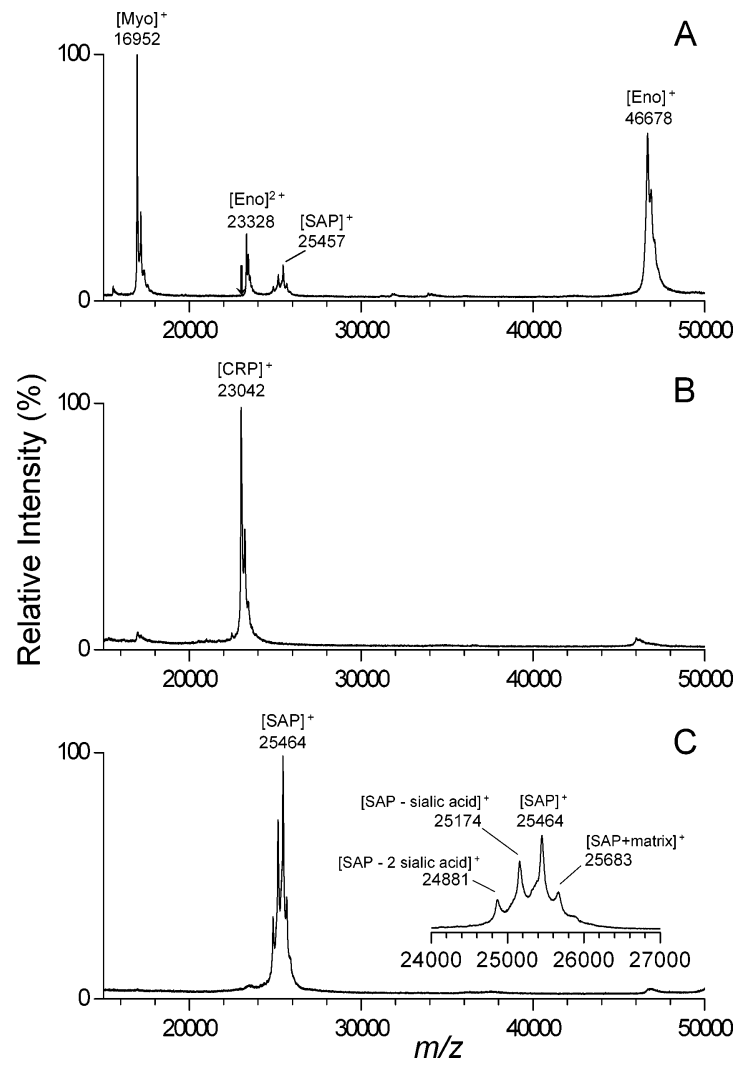

Figure 2. MALDI mass spectra of a protein mixture before $(A)$ and after using (B) anti-CRP- or (C) anti-SAP-conjugated mNPs to extract a specific protein. The protein solution $(60 \mu \mathrm{L})$ was composed of 0.5 $\mu \mathrm{M}$ myoglobin (Myo), $0.1 \mu \mathrm{M}$ C-reactive protein (CRP), $0.67 \mu \mathrm{M}$ serum amyloid $\mathrm{P}$ component (SAP), and $2.1 \mu \mathrm{M}$ enolase (Eno). The arrow in the mass spectrum of $(A)$ indicates the theoretical $\mathrm{m} / \mathrm{z}$ of CRP. The inset of $(C)$ shows detailed protein expression profiles of wild type, monosialo-, and asialo-SAP.

and sample desalting without the necessity of additional elution steps. In general, antibody - antigen interactions are strong, having dissociation constants $\left(K_{\mathrm{d}}\right)$ ranging from $10^{-7}$ to $10^{-11} \mathrm{M}$. Most antibody-antigen complexes can be dissociated at extreme $\mathrm{pH}$ (i.e., $\mathrm{pH}<2$ or $\mathrm{pH}>12$ ). The $\mathrm{pH}$ of matrix solution (SA) is typically less than 2 and thus may serve to elute the antigen bound to the antibody-conjugated mNPs.

Mass spectrometric detection is also ideal for characterizing posttranslational modifications that cannot be predicted from genomic information. The MALDI spectrum in Figure $2 \mathrm{C}$ shows a cluster of peaks corresponding to several SAP variants from the affinity extraction using anti-SAP-conjugated mNPs. The expanded view shows that mass spectrum is dominated by the mass of $25464 \pm 4 \mathrm{Da}$, consistent with the theoretical value of $25462 \mathrm{Da}$, as calculated from the known sequence. ${ }^{51}$ Accompanying the major peak were two peaks at 25174 and $24881 \mathrm{Da}$, corresponding to mass shifts of 290 and $583 \mathrm{Da}$, respectively. Within the experimental uncertainty, the shifts can be attributed to the loss of one or two sialic acid residues (each residue MW $=291) .{ }^{25} \mathrm{In}$ the linear mode, we found that the mass resolution did not deteriorate when the antigen-bound mNPs were deposited on the MALDI probe. Similarly, a mass accuracy of $0.02 \%$ could be routinely obtained by external calibration, comparable to the mass accuracy of conventional MALDI detection. Thus, the "direct"

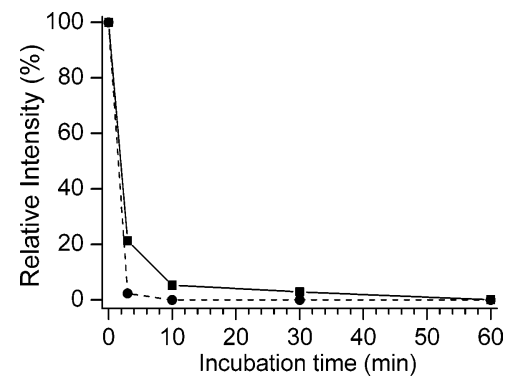

Figure 3. Effect of incubation time on antibody-antigen recognition using antibody-conjugated mNPs. To investigate the time course of antibody-antigen recognition on mNPs, $1 \mu \mathrm{L}$ of supernatant was sampled from a $60-\mu \mathrm{L}$ reaction after different incubation times. The mNPs were conjugated with: anti-SAP (squares with solid line) and anti-CRP (circles with dashed line). After incubation ( $3 \mathrm{~min}-1 \mathrm{~h}$ ), the quantities of the antigen remaining in the supernatant were detected by MALDI MS, and the peak intensities were plotted as a function of incubation time.

analysis of mNPs does not diminish the performance of the MALDI MS

Kinetic Study of the Immunoreaction. The use of antibodyconjugated mNPs significantly reduces sample handling time. In immunological assays, the incubation of antibody and antigen is often the rate-limiting step (e.g., $30 \mathrm{~min}$ to overnight for conventional ELISA). 58,59 To evaluate the efficiency of our method, we investigated the effect of incubation time on antibody-antigen recognition. After incubation of antibody-conjugated mNPs with antigen solution, the amount of remaining antigen was measured by MALDI MS. Figure 3 shows that the peak intensities, corresponding to unbound antigen (SAP) in solution, decreased dramatically as a function of incubation time over $10 \mathrm{~min}$, at which time free SAP was barely detectable (signal-to-noise ratio was $<3$ ). Significantly, maximum binding of CRP was almost completed in even shorter incubation time ( $<3 \mathrm{~min})$.

Our approach directly detected specific antibody-captured antigens by MALDI MS without using a secondary antibody or a reporter reaction. Unlike conventional immunoassays such as ELISA, for which the overall process usually requires at least 4 h, our mNP-based immunoassay can be shortened within 15-20 min. Thus, this rapid and sensitive approach may be amenable to clinical applications such as high-throughput or population screening.

Detection Sensitivity. Another advantage of the nanoprobebased immunoassay is the ability to preconcentrate the antigen from diluted medium to a small volume of mNPs. To demonstrate this concentration effect, a series of solutions with different SAP concentration $(8-160 \mathrm{nM})$ were prepared by diluting an equal quantity $(8 \mathrm{pmol})$ of SAP into different volumes. Figure 4 shows the MALDI mass spectra of extracted SAP after preconcentration using anti-SAP-conjugated mNPs. By contrast, the SAP peak was barely discernible (or not detected) when the diluted samples were analyzed by conventional MALDI, as shown in the inset of each panel.

The lower limit of detection of the approach was explored using different amounts of SAP. After affinity extraction, Figure 5 shows

(58) Fu, J. Y.; Muller, D. Calcif. Tissue Int. 1999, 64, 229-233.

(59) Wang, X.; Chen, F.; Wan, P. J.; Huang, G. J. Agric. Food Chem. 2004, 52, $7793-7797$. 


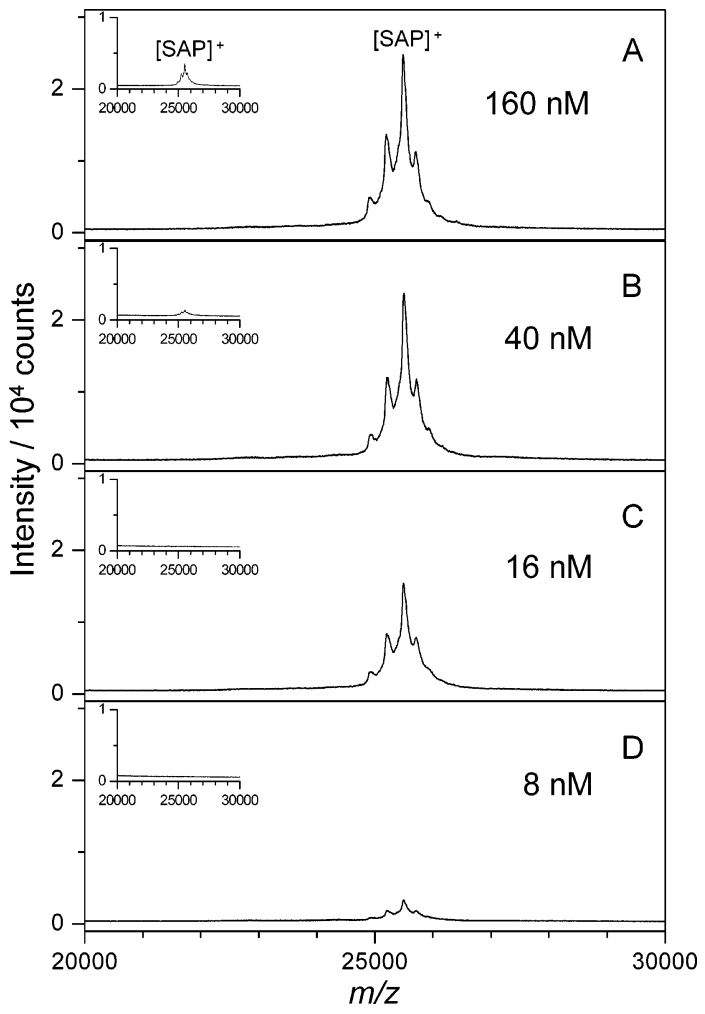

Figure 4. MALDI mass spectra of SAP extracted from diluted solution using anti-SAP-conjugated mNPs: (A) $160 \mathrm{nM} \mathrm{SAP,} 50 \mu \mathrm{L}$; (B) $40 \mathrm{nM}, 200 \mu \mathrm{L}$; (C) $16 \mathrm{nM}, 500 \mu \mathrm{L}$; (D) $8 \mathrm{nM}, 1000 \mu \mathrm{L}$. The inset of each panel shows the mass spectrum of solution prior to extraction.

the mass spectra of $60 \mu \mathrm{L}$ of SAP solution after a series of dilutions ranging from $54 \mu \mathrm{g} / \mathrm{mL}$ to $16 \mathrm{ng} / \mathrm{mL}(1.9 \mu \mathrm{M}-0.6 \mathrm{nM})$. The SAP signals decrease progressively with decreasing concentration. Strong peak intensities were observed in all spectra except that for the $0.6 \mathrm{nM}$ solution, which had a signal-to-noise ratio of 3 . Theoretically, the sensitivity of the current approach depends on the MALDI MS detection sensitivity and the efficiency of affinity extraction. Assuming full recovery of all the SAP present in the $60 \mu \mathrm{L}$ of diluted solution ( $0.6 \mathrm{nM}$ in Figure $5 \mathrm{E})$, the absolute detection limit is estimated to be $36 \mathrm{fmol}$, which is comparable to the detection limit by direct deposition of SAP onto the MALDI probe (data not shown). It is noteworthy that SAP and CRP levels in sera from healthy individuals were about $1.6 \mu \mathrm{M}$ and $40 \mathrm{nM}$, respectively. ${ }^{49,50}$ Considering the presented limit of detection in the subnanomolar level $(0.6 \mathrm{nM})$, our nanoscale immunoassay is theoretically capable, with reasonable enrichment, of detecting both of these targeted proteins in plasma.

Detection of CRP and SAP from Human Plasma. It is well recognized that the human plasma proteomics holds the promise of a revolution in both disease diagnosis and therapeutic aspects. ${ }^{5}$ However, human plasma is a very complex mixture of proteins having a wide and dynamic range of abundance of more than $10^{12}$. Indeed, 22 proteins constitute $\sim 99 \%$ of the protein content in plasma, with the remaining $1 \%$ comprising low-abundance proteins that are of great interest as potential biomarkers. ${ }^{60}$ Thus, we investigated whether the nanoprobe-based platform can be used to directly detect low-level proteins in human plasma.

(60) Tirumalai, R. S.; Chan, K. C.; Prieto, D. A.; Issaq, H. J.; Conrads, T. P.; Veenstra, T. D. Mol. Cell. Proteomics 2003, 2, 1096-1103.

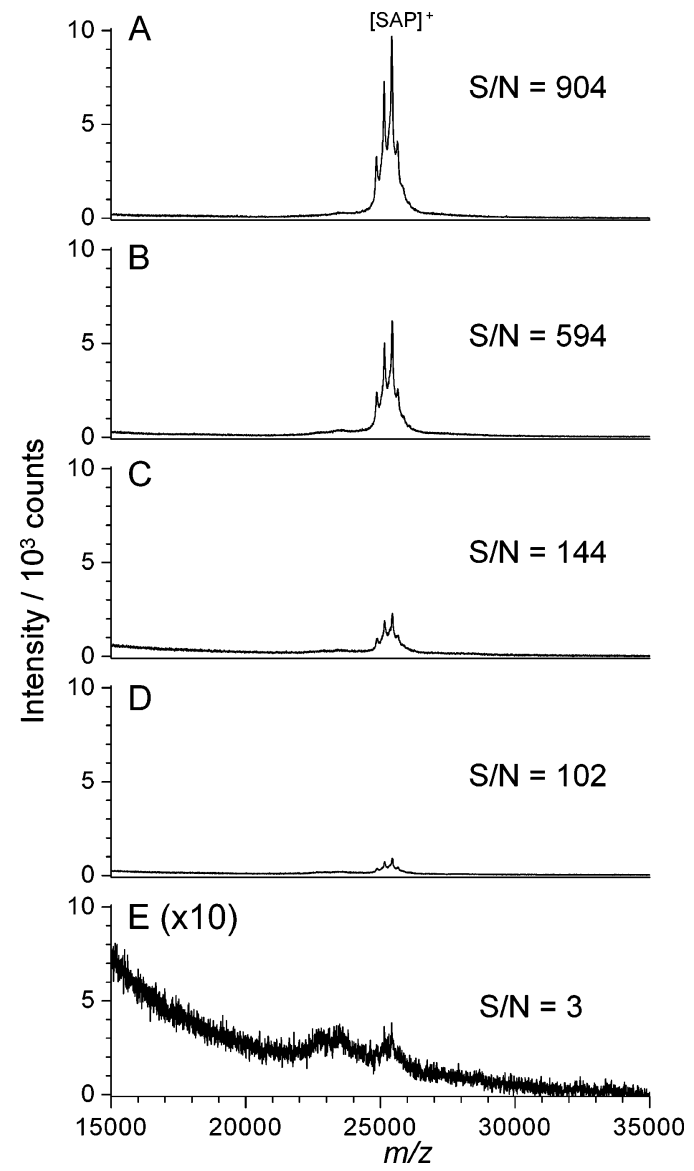

Figure 5. MALDI mass spectra of SAP extracted from $60 \mu \mathrm{L}$ of protein solution of (A) $1.9 \mu \mathrm{M}$, (B) $80 \mathrm{nM}$, (C) $15 \mathrm{nM}$, (D) $3 \mathrm{nM}$, and (E) $0.6 \mathrm{nM}$, using anti-SAP-conjugated mNPs.

To evaluate the specificity of our immunoassay, $5 \mu \mathrm{L}$ of plasma from a healthy subject was incubated sequentially with anti-SAPand with anti-CRP-conjugated mNPs for affinity extraction of SAP and CRP, respectively. Prior to immunoaffinity extraction, no protein profile could be obtained from the stock plasma sample due to the interference of the salt and other plasma components. We thus diluted the plasma sample 200-fold to reduce the salt concentration and subjected it to the analysis. The protein profile in Figure 6A shows the commonly observed abundant plasma proteins, including human serum albumin (HSA), apolipoprotein A-I (ApoA-I), hemoglobin $\alpha$-chain (Hb-A), hemoglobin $\beta$-chain (Hb-B), and transthyretin (TTR). After immunoaffinity extraction, SAP was detected with concomitant depletion of other proteins of higher concentration (Figure 6B). Similarly, Figure $6 \mathrm{C}$ shows an apparent peak for CRP, even though the level of this protein is 40-fold significantly lower than that of SAP in healthy individuals. Although the analysis showed minor peaks due to nonspecific binding of other plasma protein, they did not interfere with the unambiguous identification of CRP and SAP.

Comparison between Nanoscale Particles and Microscale Particles. Recently, absorption of peptides/proteins onto microscale, reversed-phase magnetic particles was used to preconcentrate a dilute, contaminated sample for peptide mass mapping via MALDI analysis. ${ }^{61-63}$ Approaches that conjugated antibody to

(61) Doucette, A.; Craft, D.; Li, L. Anal. Chem. 2000, 72, 3355-3362. (62) Yaneva, M.; Tempst, P. Anal. Chem. 2003, 75, 6437-6448. 

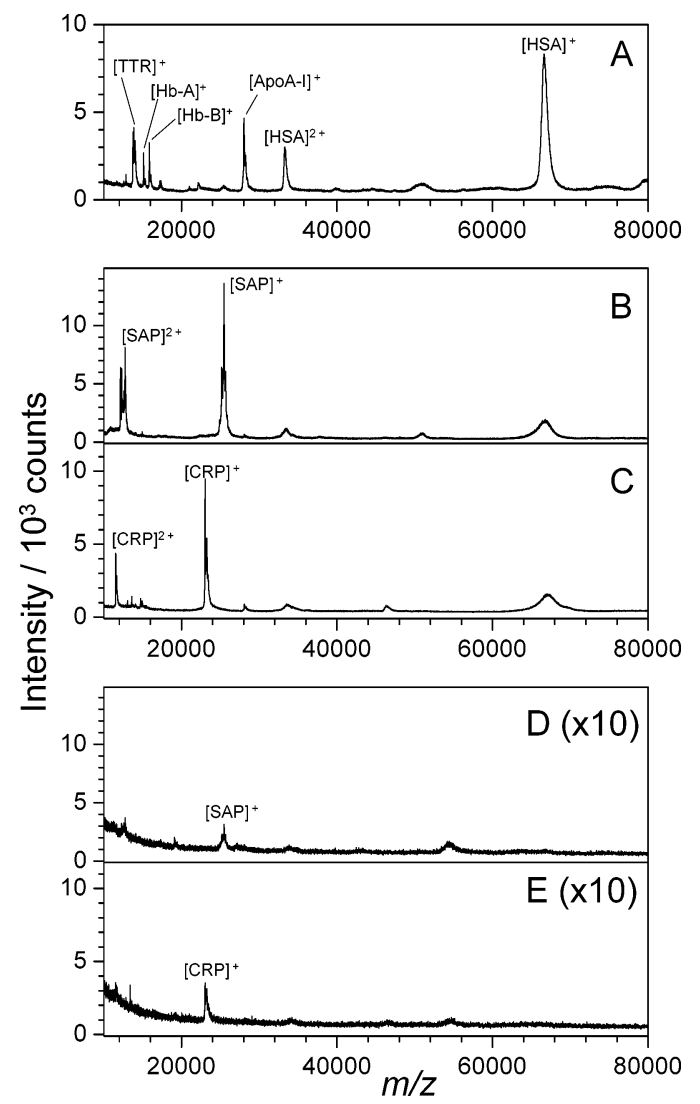

Figure 6. Comparison of affinity extraction between antibodyconjugated magnetic nanoparticles and magnetic microbeads. (A) MALDI mass spectrum of 200 -fold-diluted plasma. High-abundance proteins were so dominant that the signals from SAP or CRP were buried in the spectrum. Representative mass spectra of (B) SAP and (C) CRP were obtained after extraction using antibody-conjugated mNPs. Spectra for antibody-conjugated microbeads are shown in (D) SAP and (E) CRP.

microbeads have been reported to capture antigens of interest, yet these direct covalent conjugation procedures suffer from high background from nonspecific binding and low signal-to-noise ratio. ${ }^{64}$ Thus, we compared microscale particle and nanoscale particle with regard to extraction efficiency and detection specificity. Anti-SAP antibody was conjugated to commercially available aminated magnetic microbeads $(2.8 \mu \mathrm{m})$ by the same modification process used for aminosilane mNPs. The amounts of immobilized anti-SAP antibodies were determined to be 47 and $26 \mu \mathrm{g} / \mathrm{mg}$ for mNPs and microbeads, respectively, using the BCA protein assay. Thus, to ensure that equal amounts of antibody were used for antigen capture, we used a 1:1.8 volume ratio of nanoparticle/ microbeads in parallel immunoassays.

As shown in Figure 6D and E, the signal intensity and signalto-noise ratio were dramatically reduced in the mass spectrum of microbead-captured SAP and CRP compared with the nanoparticle experiments (Figure $6 \mathrm{~B}$ and $\mathrm{C}$ ). These results indicate that $\mathrm{mNPs}$ afford better affinity extraction of the targeted protein, thereby improving the detection limit. ${ }^{40}$ The fact that the same number of

(63) Villanueva, J.; Philip, J.; Entenberg, D.; Chaparro, C. A.; Tanwar, M. K.; Holland, E. C.; Tempst, P. Anal. Chem. 2004, 76, 1560-1570.

(64) Peter, J. F.; Tomer, K. B. Anal. Chem. 2001, 73, 4012-4019.

(65) Soukka, T.; Paukkunen, J.; Harma, H.; Lonnberg, S.; Lindroos, H.; Lovgren, T. Clin. Chem. 2001, 47, 1269-1278.

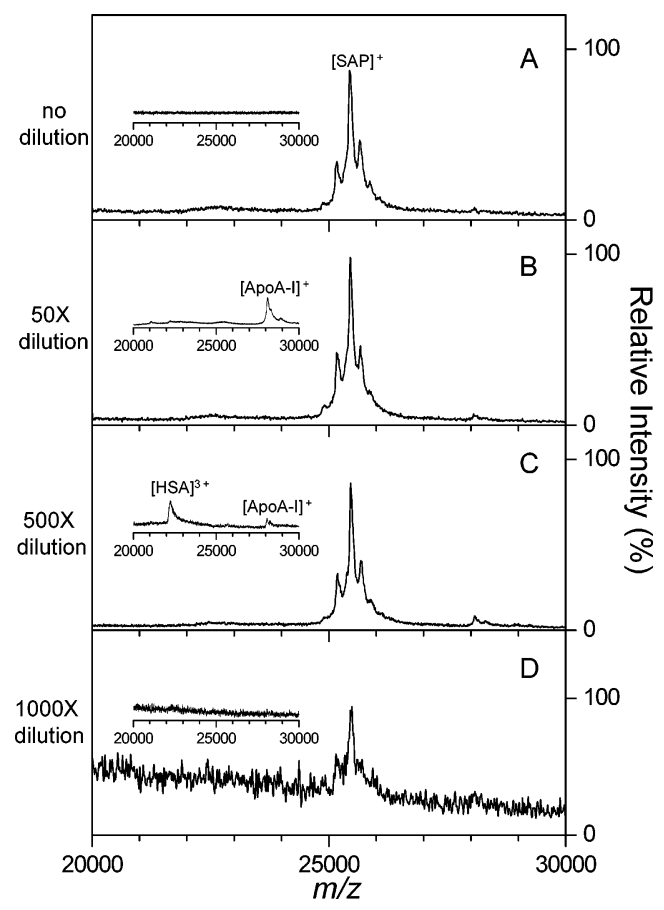

Figure 7. MALDI mass spectra of SAP extracted from $1 \mu \mathrm{L}$ of human plasma, either undiluted (A), diluted 50-fold (B), diluted 500fold (C), or diluted 1000 -fold (D), using anti-SAP-conjugated mNPs extraction. The inset of each panel shows the mass spectrum of solution prior to extraction.

antibodies was immobilized on the surfaces of the nanoscale and microscale particles suggests that the multivalent interaction ${ }^{70}$ between the targeted antigen and antibody-conjugated nanoparticles yields superior sensitivity. The superior efficiency of nanoscale particle could be attributed to its large surface area-to-volume ratio and good reaction homogeneity in suspension array. Additionally, compared with the slightly reduced resolution using microbeads ${ }^{66}$ both mass resolution and profile were maintained using mNPs, without apparent peak broadening, mass shift, or both.

Concentration Effect for Plasma Protein Profiling. Affinity extraction of targeted antigens using antibody-conjugated mNPs not only isolates but also preconcentrates low-level antigens onto the nanoprobe. To quantify this concentration effect, equal amounts of plasma ( $1 \mu \mathrm{L}$ from each subject) were diluted 50-, 500-, and 1000-fold in PBS and analyzed using our immunoassay The plasma SAP level of this subject had been rigorously determined to be $45.4 \pm 3.2 \mathrm{mg} / \mathrm{L}(1.8 \mu \mathrm{M}) .{ }^{67}$ Figure 7 shows the MALDI mass spectra of SAP extracted from each diluted sample. Incubation of the diluted plasma samples with the antibody-conjugated mNPs resulted in selective concentration of SAP, as demonstrated by the similar mass spectra profiles up to 500-fold dilution (Figure $7 \mathrm{~A}-\mathrm{C})$. In the 1000-fold diluted sample (1.8 nM SAP), however, the captured antigen showed significantly lower intensity in the mass spectrum (Figure 7D). We speculate that this decreased recovery was a consequence of incomplete recovery of the mNPs from the curved wall of the microcentrifuge tube during the washing steps, due to the large initial volume. These results

(66) Papac, D. I.; Hoyes, J.; Tomer, K. B. Anal. Chem. 1994, 66, 6, 2609-2613. (67) Chen, S. H. Affinity Mass Spectrometry for Targeted Proteomics. Master's Thesis, National Taiwan University, Taipei, Taiwan, 2004. 


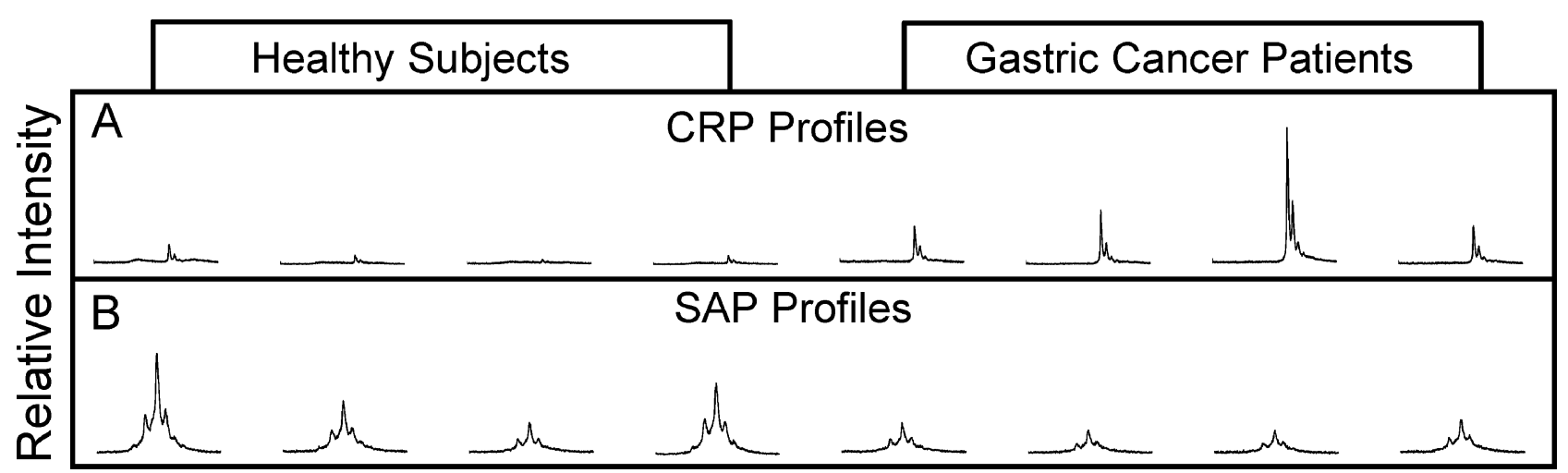

Figure 8. Screening of human plasma from healthy individuals and patients with gastric cancer using the nanoparticle-based mass spectrometric immunoassay. The MALDI mass spectra of CRP, in the $\mathrm{m} / \mathrm{z}$ range of $20000-25000$, are shown in panel A. The MALDI mass spectra of SAP, in the $m / z$ range of $24000-27000$, are shown in panel $B$.

demonstrate that $1 \mu \mathrm{L}$ of plasma is sufficient to unambiguously identify an antigen of interest using our immunoassay. Despite the decreased recovery at 1000-fold dilution, the detection sensitivity (estimated to be $1.8 \mathrm{nM}$ ) was comparable to the sensitivity using a protein standard (SAP), demonstrating that the assay is refractory to the presence of highly abundant nonantigenic proteins, salts, and buffers in plasma.

Analysis of Clinical Samples. We assessed the performance of our immunoassay using authentic clinical samples-plasma from four healthy individuals and four patients with gastric cancer (Figure 8). We detected CRP and SAP in all the healthy individuals, despite the fact that the levels of a few of them were below the detection limit of ELISA $(<0.159 \mathrm{mg} / \mathrm{L}) .^{53}$ The measured intensities for CRP were considerably higher in the gastric cancer patient samples than in the healthy control samples. By contrast, the SAP levels in the patients were lower than those in the healthy individuals. These observed differences in protein levels are consistent with the differential protein profiles of gastric cancer patients as assessed by comparative proteomic approaches. ${ }^{68,69}$ We note that the ion intensity measured by the nanoprobe-based assay using a $20-\mu \mathrm{L}$ sample correlated with the concentration measured by ELISA, suggesting that our immunoassay shows promise for quantitative protein profiling. Whether the trend demonstrated by our approach is statistically significant, more samples from patients are required for further evaluation, an aspect that we are currently investigating.

\section{CONCLUDING REMARKS}

We have shown that covalent conjugation of antibodies to mNPs yields a stable affinity probe for mass spectrometric immunoassay, providing simultaneous isolation and preconcentration of targeted proteins from unfractionated human plasma. Mass spectrometric detection allows not only protein profiling but

(68) Jang, J. S.; Cho, H. Y.; Lee, Y. J.; Ha, W. S.; Kim, H. W. Oncol. Res. 2004 , 14, 491-499.

(69) Solakidi, S.; Dessypris, A.; Stathopoulos, G. P.; Androulakis, G.; Sekeris, C. E. Clin. Biochem. 2004, 37, 56-60.

(70) Gestwicki, J. E.; Cairo, C. W.; Strong, L. E.; Oetjen, K. A.; Kiessling, L. L. J. Am. Chem. Soc. 2002, 124, 14922-14933. also screening of glycan structure mircroheterogeneity of targeted antigens. The assay achieved subnanomolar $\left(10^{-9}-10^{-10} \mathrm{M}\right)$ limits of detection and showed good extraction efficiency for plasma protein diluted up to 500-fold. With the current detection sensitivity, the SAP and CRP levels in healthy human plasma could be analyzed unambiguously. Our results indicate that the approach can be used to rapidly screen relatively low-level targeted proteins in complex mixtures containing other high-abundance proteins such as serum albumin. Compared with commercially available microscale particles, antibody-conjugated mNPs exhibited significantly better extraction efficiency and specificity.

The strength of this approach was manifold. First, it is a very economical assay, with each experiment costing about \$0.30 U.S. Moreover, from sample preparation to mass spectrometric analysis, this immunoassay can be completed in less than $20 \mathrm{~min}$, and the approach potentially may be adapted to high-throughput nanoarray formats. Furthermore, the assay has high sensitivity that requires a sample size of only $1 \mu \mathrm{L}$ of plasma from healthy individuals or patients to generate the targeted protein profiles. In combination with the ongoing quantitative analysis of plasma protein profiling, this nanoprobe-based immunoassay holds great potential for the early diagnosis of disease, inflammatory events, and eventually cancers. The specificity, speed, and flexibility of the nanoprobe-based affinity assay can easily be adapted for the detection of other class-specific proteins in proteomic applications.

\section{ACKNOWLEDGMENT}

We acknowledge financial support from Academia Sinica and the National Science Council, Taiwan. We greatly appreciate the ELISA analyses performed by Mr. Li-Li Wen at En Chu Kong Hospital and the use of MALDI-TOF MS at the Institute of Biomedical Sciences, Academia Sinica, Taiwan.

Received for review April 18, 2005. Accepted June 8, 2005.

AC0506550 